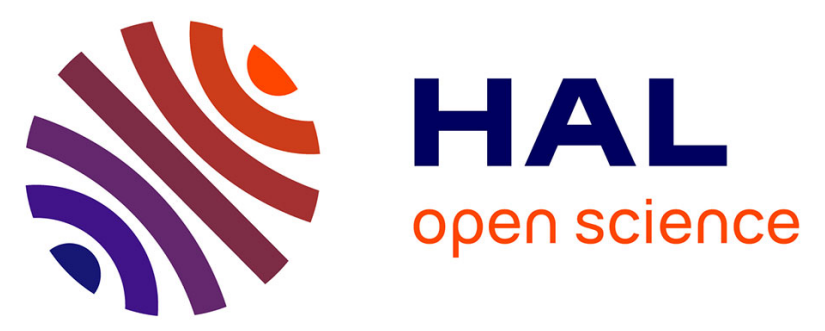

\title{
Imaging orbitals with attosecond and Ångström resolutions: toward attochemistry? Imaging orbitals with attosecond and Ångström resolutions: toward attochemistry?
}

Pascal Salières, A Maquet, Stefan Haessler, J Caillat, R Taïeb

\section{To cite this version:}

Pascal Salières, A Maquet, Stefan Haessler, J Caillat, R Taïeb. Imaging orbitals with attosecond and Ångström resolutions: toward attochemistry? Imaging orbitals with attosecond and Ångström resolutions: toward attochemistry?. Reports on Progress in Physics, 2012, 75, pp.062401. 10.1088/00344885/75/6/062401. hal-01164756

\section{HAL Id: hal-01164756 \\ https://hal-ensta-paris.archives-ouvertes.fr/hal-01164756}

Submitted on 18 Jun 2015

HAL is a multi-disciplinary open access archive for the deposit and dissemination of scientific research documents, whether they are published or not. The documents may come from teaching and research institutions in France or abroad, or from public or private research centers.
L'archive ouverte pluridisciplinaire HAL, est destinée au dépôt et à la diffusion de documents scientifiques de niveau recherche, publiés ou non, émanant des établissements d'enseignement et de recherche français ou étrangers, des laboratoires publics ou privés. 
31 January 2012

\title{
Imaging orbitals with attosecond and Ångström resolutions: toward attochemistry?
}

\author{
P. Salières ${ }^{1}$, A. Maquet ${ }^{2}$, S. Haessler ${ }^{3}$, J. Caillat ${ }^{2}$ and R. Taïeb ${ }^{2}$ \\ ${ }^{1}$ CEA-Saclay, IRAMIS, Service des Photons, Atomes et Molécules, 91191 \\ Gif-sur-Yvette, France \\ ${ }^{2}$ UPMC Univ. Paris 6, UMR 7614, Laboratoire de Chimie-Matière et Rayonnement, \\ 11 rue Pierre et Marie Curie, 75231 Paris Cedex 05, France \\ ${ }^{3}$ Photonics Institute, Vienna University of Technology, Gusshausstrasse 27/387, 1040 \\ Vienna, Austria \\ E-mail: pascal.salieres@cea.fr
}

\begin{abstract}
.
The recently developed attosecond light sources are making possible the investigation of ultrafast processes in matter with unprecedented time resolution. It has been proposed that the very mechanism underlying the attosecond emission allows for imaging the valence orbitals with Ångström space resolution. This controversial idea together with the possibility to combine attosecond and Ångström resolutions in the same measurements have become a hot topic in strong-field science. Indeed, this could provide a new way to image the evolution of the molecular electron cloud during, e.g., a chemical reaction in "real time". Here we review both the experimental and theoretical challenges raised by the implementation of these prospects. In particular, we show how the valence orbital structure is encoded in the spectral phase of the recombination dipole moment calculated for Coulomb scattering states, which allows a tomographic reconstruction of the orbital using first-order corrections to the plane-wave approach. The possibility to disentangle multi-channel contributions to the attosecond emission is discussed as well as the necessary compromise between the temporal and spatial resolutions.
\end{abstract}

Submitted to: Rep. Prog. Phys. 


\section{Introduction}

The recent advent of attosecond sources of extreme ultraviolet (XUV) and soft x-ray radiation is opening wide perspectives for probing dynamics in matter at the natural timescale of electron motion, i.e. the atomic unit of time $\left(1 \mathrm{a} . \mathrm{u} . \approx 24 \times 10^{-18} \mathrm{~s}=24\right.$ attoseconds) [1]. It was recently realized that these same sources could also provide Ångström-resolved images of electron wavepackets in molecules [2-4]. The combination of these extreme spatial and temporal resolutions may fulfill the dream of imaging in real time the most fundamental processes occurring in physics, chemistry or biology [5]. Understanding these processes is of the utmost importance for future developments in science and technology as it is the key for controlling them.

These attosecond light sources are based on the spectacular phenomenon of highorder harmonic generation ( $\mathrm{HHG})[6,7]$ that results from the strongly nonlinear interaction of intense infra-red (IR) laser pulses with atomic or molecular gases. In spite of its apparent complexity, the generating process can be described by a fairly intuitive semi-classical three-step model $[8,9]$ : the strong laser field tunnel ionizes the molecules or atoms of the target gas, releasing an electron wavepacket (EWP) that is subsequently accelerated by the oscillating field and driven back to its parent ionic core where it recombines, emitting an attosecond burst of coherent soft x-rays. In a multicycle laser field, this process is repeated every half-cycle resulting in the emission of a train of attosecond pulses, the spectrum of which is composed of the high order harmonics of the generating IR field. This very simple model not only succeeds in explaining the main properties of HHG [10] but also lies at the heart of its use in many attosecond spectroscopy schemes [1].

The first demonstration of the generation of attosecond pulses through HHG in $2001[11,12]$ suddenly made possible a number of new investigations of dynamics in matter. The applications can be grouped in two categories. The first one concerns "conventional" pump-probe experiments, hereafter referred to as "direct" schemes, where the attosecond bunch of photons is used to excite or probe ultrafast dynamics in another system - a direct extension of femtosecond spectroscopy and femtochemistry [13] to the attosecond domain. A number of field-driven [14-17] and intrinsic [18-24] processes with attosecond dynamics have already been studied in atoms, molecules and solids using direct schemes. In the second category of experiments, the harmonic signal is analyzed to retrieve temporal and structural insight on the generating system itself. In such "self-probing" schemes, the recolliding electron wavepacket plays the role of a probe that encodes information on the generating molecule in the emitted radiation. It was long considered a theoretical challenge to extract relevant information out of such a highly nonlinear optical spectroscopy due to the complicated underlying physics. However, the spectacular simplifications brought about by the semiclassical model were tested and (partly) validated by a number of experimental and theoretical studies [25], providing direct information on the radiating system with unprecedented temporal and spatial resolutions. 
Indeed, advanced characterization of the attosecond soft x-ray emission gives precise information on both the structure and dynamics of the target gas. The reason is that the recolliding EWP associated with the observed photon emission has a duration of a few hundred attoseconds and a de Broglie wavelength of the order of molecular internuclear distances $(\approx 1 \AA)$. It is thus extremely sensitive to ultrafast nuclear or electronic dynamics occurring inside its parent molecular ion: Rotational [26-28], vibrational [29$32]$ and electronic $[3,33,34]$ dynamics have already been successfully studied using this self-probing scheme. For instance, it was found that a change in bond length of $\approx 1 \%$ may result in a change in high-harmonic signal of $\approx 10 \%$, allowing Raman vibrations to be observed in $\mathrm{SF}_{6}[30]$ or ultrafast proton rearrangements to be measured in $\mathrm{H}_{2}^{+}$ and $\mathrm{CH}_{4}^{+}$with 100 as resolution [29,32]. Besides, multielectron dynamics also leave characteristic fingerprints in the HHG emission, which allows studying multichannel tunnel ionization with attosecond resolution [3, 33, 34].

Another, most fascinating, perspective opened by the self-probing scheme is the possibility not-only to time-resolve attosecond intra-molecular dynamics but also to spatially image the wavefunction of the radiating system with Angström resolution, as first proposed by Itatani et al. [2]. The basic idea is that, during the HHG process, the ionic core is probed by the laser-driven rescattering electron in a well defined direction given by the laser polarization axis. This makes HHG strongly dependent on the molecular orientation with respect to the laser polarization. It was suggested that, via a tomographic analysis of the harmonic signal obtained at different relative orientations, one could reconstruct an image of the orbital from which the electron was released. The intrinsic coherence of the process, more precisely the coherence of the EWP formed by a combination of scattering states and the initial bound state, in principle gives access not only to the modulus of the radiating dipole but also to its phase, and therefore to the electron wavefunction, in amplitude and phase (up to a global constant). This came somewhat as a surprise since a paradigm of quantum mechanics is that a wavefunction is merely a mathematical construction and that only electron densities are "observable" quantities. For a discussion on the feasibility of wavefunction "measurements", see [35]. Concerning the access to the wavefunction phase, it is provided by interferometric intensity measurements made possible by the coherence of the process, as mentioned above - in contrast to photoemission spectroscopy [36] and scanning tunnelling microscopy $[37,38]$ that give access only to electron densities.

An important property of $\mathrm{HHG}$ is that, since tunnel ionization depends exponentially on the binding energy, the emission process intrinsically selects the higher lying populated electronic levels, and is therefore particularly sensitive to the stucture of the valence electronic states, which are those participating in chemical reactions. It has thus been suggested that this could provide a new way to image the evolution of the molecular electron cloud during a chemical change, in "real time" and with $\AA$ resolution. Due to its high potential and implications, this possibility to monitor orbitals and dynamics of chemical reactions on an attosecond timescale has become a "burning question" in high-field science, motivating a large number of studies, the main outcome 
of which being that there are still several major issues at stake before reaching this goal. Here we shall address a few of them, from both the experimental and theoretical point of views, and discuss the problem in the light of recent works [3, 4, 39]. After a brief presentation of the principles of tomographic orbital imaging in Section 2, we shall review the experimental techniques and challenges raised by its implementation in Section 3. We shall then discuss the type of information that can be accessed from the HHG process on three levels: i) the recombination dipole moment in Section 4; ii) the orbital reconstruction in Section 5; iii) the dynamics occurring in the radiating system in Section 6. We shall review the different challenges raised by each level in view to fulfill the dream of imaging changing orbitals during a chemical reaction.

\section{Principles of tomographic orbital imaging}

This section briefly presents the theoretical basis for the orbital tomography; a more detailed account can be found in the tutorial [25]. The general theoretical framework is provided by the quantum description of HHG for a fixed-in-space molecule interacting with the strong laser electric field. Retrieving a complete tomographic image in principle requires to probe the molecule from all orientations $\Omega=(\theta, \phi)$, defined between the laser polarization and a chosen molecular axis $(O x)$. In the following, we will however restrict our procedure to $2 \mathrm{D}$ imaging, by varying a single angle, $\theta$, in one of the molecular planes, denoted $(x O y)$ (see Fig. 1). The reconstructed image will turn out to be the projection of the 3D orbital on this plane. The main reason for this is that we will illustrate the procedure by applying it to the nitrogen molecule, for which the $2 \mathrm{D}$ projected reconstruction already contains all the structural information - as for any object with (assumed) cylindrical symmetry.

From the solution $\left|\psi_{\theta}(t)\right\rangle$ of the Time-Dependent Schrödinger Equation (TDSE) for the molecule's active electron(s) at a given orientation $\theta$, one can express the dipole moment:

$$
\overrightarrow{\mathcal{D}}(t, \theta)=-e\left\langle\psi_{\theta}(t)|\hat{\mathbf{r}}| \psi_{\theta}(t)\right\rangle,
$$

where $e$ is the electron charge. The acceleration of this dipole moment is the source term inserted in the Maxwell's equations (wave equation) for calculating the coherent growth of the harmonic emission [40]. Under proper phase matching conditions, the macroscopic harmonic field $E_{X U V}(\omega, \theta)$ at frequency $\omega$ is then determined by the $\omega$ component of the Fourier transform of the single-molecule dipole acceleration:

$$
\vec{D}(\omega, \theta) \approx \mathcal{F}_{t \rightarrow \omega}\left[-\frac{\mathrm{d}^{2}}{\mathrm{~d} t^{2}} \overrightarrow{\mathcal{D}}(t, \theta)\right] \approx \omega^{2} \mathcal{F}_{t \rightarrow \omega}[\overrightarrow{\mathcal{D}}(t, \theta)] .
$$

Using an ansatz for $\left|\psi_{\theta}(t)\right\rangle$ based on the strong-field approximation (SFA) [10], the quantum version of the three-step model results in a factorized expression for the complex valued dipole [41-44]:

$$
\vec{D}(\omega, \theta)=\kappa(\vec{k}) a(\vec{k}) \vec{d}(\vec{k})
$$




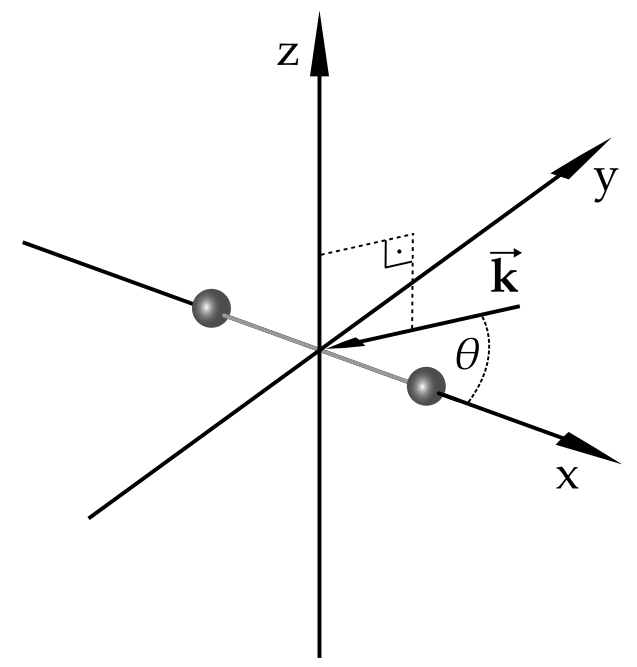

Figure 1. Reference frame for a diatomic molecule in the self-probing scheme: $(x O y)$ is defined as the plane containing both the molecular axis $(O x)$ and the laser polarization direction, along which the freed EWP recollides with momentum $\vec{k}$. The probing orientation with respect to $(O x)$ is $\theta$.

where each factor accounts for one of the three steps of the semi classical model. The first factor $\kappa(\vec{k})$ is the (in general complex-valued) $\theta$-dependent tunnel ionization amplitude, $a(\vec{k})$ accounts for the propagation of the laser-driven EWP in the continuum and $\vec{d}(\vec{k})=\left\langle\varphi_{0}|\hat{\mathbf{r}}| \varphi_{\vec{k}}\right\rangle$ is the (length-form) dipole recombination matrix element between the initial orbital $\varphi_{0}$ (the Dyson orbital in a polyelectronic system, see below) and the continuum wave function $\varphi_{\vec{k}}$. The asymptotic momentum of the recolliding electron, $\vec{k}$, has its direction parallel to the laser polarization, and a modulus related to $\omega$ by the energy conservation at recombination:

$$
\hbar \omega=\hbar^{2} k^{2} / 2 m+I_{p}
$$

with $I_{p}$, the ionization potential of the system and $m$ the electron mass. Note that the SFA predicts the EWP associated to a given frequency $\omega$ to mostly follow two semi-classical trajectories during its excursion in the continuum: the so-called short and long trajectories [41]. The above factorization assumes that the contributions from a single class of trajectories were selected (see Section 3.2 for the trajectory filtering in experiments).

The complex valued recombination dipole matrix element $\vec{d}(\vec{k})$ is the one needed to retrieve an image of the molecular orbital. In order to extract this dipole from the measured harmonic emission, one needs to determine the first two factors: the tunnel ionization amplitude $\kappa(\vec{k})$ and the continuum amplitude $a(\vec{k})$ which are not easily accessible from either theory or experiment. However, as suggested initially in [2], one can circumvent this difficulty by calibrating the harmonic measurements with the HHG of a reference system (typically a rare gas atom) with same $I_{p}$. Indeed, given the exponential dependence on $I_{p}$ of tunnel ionization, species with the same ionization energy are ionized at the same time during the laser cycle, i.e. with the same potential 
barrier width. The $\vec{k}$-dependence of their tunnel ionization amplitudes can thus be assumed to be equal. Moreover, the factors $a(\vec{k})$, associated with the motion of the laser-driven continuum electron wave packets, are assumed to be essentially the same in the molecule considered and in the reference system when the driving laser field is the same. Then, if the recombination dipole matrix element $d_{\text {ref }}(\vec{k})$ of the latter is known, e.g. from theory, the molecular dipole is obtained from the ratio of the measured signals:

$$
\vec{d}(\vec{k})=\frac{1}{\eta(\theta)} \frac{\vec{D}(\omega, \theta)}{D_{\text {ref }}(\omega)} d_{\text {ref }}(\vec{k})
$$

where the label ref is attached to the quantities obtained in the reference system for the same laser parameters. The complex valued $\eta(\theta)$ stands for the $\theta$-dependence of the tunnel ionization amplitude in the molecule, left after the $\vec{k}$-dependence has been calibrated for. Note that the reference dipoles $D_{\text {ref }}$ and $d_{\text {ref }}$ are scalars: the recombination dipole for an atom and hence the corresponding harmonic dipole are always polarized parallel to the electron recollision direction, and we divide by this component $\| \vec{k}$.

Thus, the components of the complex molecular "free-bound" recombination dipole vector can be determined in the molecular frame through the measurements of the amplitudes, phases and polarization of the harmonics emitted by the molecule and reference atom gases. The corresponding "bound-free" ionization dipole has been measured in a number of studies through XUV photoionization (PI) spectroscopy, using, e.g. synchrotron radiation. The HHG self-probing scheme brings two important advances: i) it measures the dipole during the HHG emission process, which is confined to the femtosecond/attosecond timescale; this high temporal resolution allows the study of fast dynamical processes occurring in the molecules; ii) HHG gives access to the spectral phase of the dipole which is not accessible in PI experiments. The latter property allowing the retrieval of advanced spatial information on the molecule is at the heart of the tomographic procedure.

Going further in the analysis is made possible by a basic assumption of the SFA [10], according to which the EWP dynamics in the continuum is only determined by the laser field with no influence of the ionic core. The scattering waves are then approximated by plane waves, i.e. $\varphi_{\vec{k}}(\vec{r}) \approx e^{i \vec{k} \cdot \vec{r}}$. The $q$-component $(q=x, y)$ of the recombination dipole in the molecular frame then writes $(z$ is the laser propagation direction, such that $\left.k_{z}=0\right)$ :

$$
\begin{aligned}
d_{q}(\vec{k})=\left\langle\varphi_{0}|\hat{q}| \varphi_{\vec{k}}\right\rangle & \approx \int \varphi_{0}^{\star}(\vec{r}) q e^{i \vec{k} \cdot \vec{r}} d \vec{r} \\
& =\iint\left[q \int \varphi_{0}^{\star}(x, y, z) \mathrm{d} z\right] e^{i\left(k_{x} x+k_{y} y\right)} \mathrm{d} x \mathrm{~d} y
\end{aligned}
$$

Each component of $\vec{d}$ thus contains the spatial Fourier transform of

$$
q \tilde{\varphi_{0}}(x, y)=q \int \varphi_{0}^{\star}(x, y, z) \mathrm{d} z,
$$

i.e. $q$ times the initial orbital projected onto the plane perpendicular to the laser propagation direction. This implies that an orbital odd in $z$ will not contribute. For an 
even orbital with a known symmetry $(\sigma, \pi \ldots)$ around some axis in the $x$ - $y$-plane, this projection contains the complete information.

Measuring $d_{q}$ for one recollision angle $\theta$ thus yields data points in Fourier space of the object $q \tilde{\varphi}_{0}(x, y)$. These points, with coordinates $\left(k_{x}, k_{y}\right)$, all lie on a line along the recollision angle, $\theta$, at distances from the origin equal to the electron wave numbers $k$ related to the harmonic frequencies by energy conservation (Eq. 4). A whole spectrum of XUV frequencies, mapped onto points $\left(k_{x}, k_{y}\right)$ consequently yields a slice through one quadrant of Fourier space. A discretized collection of data points is recovered slice-byslice over the Fourier space by repeating the measurement for a sample of $\theta$-values. The inverse 2D Fourier transform $\mathcal{F}_{\vec{k} \rightarrow \vec{r}}$, applied to the so-obtained data, thus yields $q \tilde{\varphi}_{0}(x, y)$ in real space, and the sought-for molecular orbital (projection) can be reconstructed as

$$
\tilde{\varphi}_{0}^{q}(x, y)=\frac{\mathcal{F}_{\vec{k} \rightarrow \vec{r}}\left[d_{q}\left(k_{x}, k_{y}\right)\right]}{q} .
$$

An explicit, discretized version of this equation is given as equation 2 in ref. [3]. Note that from both dipole matrix element components, $x$ and $y$, i.e. parallel and perpendicular to the molecular axis, the same orbital can in principle be reconstructed.

The same scheme can be written based on the velocity form of the recombination dipole matrix element $d_{q}^{\mathrm{V}}\left(k_{x}, k_{y}\right)$. The orbital can then be obtained using:

$$
\tilde{\varphi}_{0}^{q}(x, y)=\mathcal{F}_{\vec{k} \rightarrow \vec{r}}\left[\frac{d_{q}^{\mathrm{V}}\left(k_{x}, k_{y}\right)}{k_{q}}\right] .
$$

Due to the plane-wave approximation that breaks the gauge invariance, formulations (8) and (9) are not equivalent and may give different results. Which is the most appropriate one is still hotly debated in the community and so far depends on the case studied [45]. While the fundamental justifications in favor of a form or the other are still unclear, there may be technical reasons to prefer one or the other: if $\tilde{\varphi}_{0}$ has a nodal plane containing the $x$ - or $y$-axis, one will run into numerical problems when dividing by $x$ or $y$ in (8).

It is straightforward to generalize the principle of the procedure, presented here for $2 \mathrm{D}$ reconstructions, to retrieve the full 3D orbital. The dipole needs to be sampled in $3 \mathrm{D}$ - either by performing the measurements also outside the $(x O y)$ plane, or by extrapolating the 2D measurements to 3D applying assumed symmetries.

The orbital imaging procedure raises a number of important questions concerning its applicability to real-world molecular systems. These questions can be divided in two classes regarding either the experimental conditions, to assess the precision of the measurements, or the theoretical aspects, to appraise its range of validity.

\section{Experimental techniques and challenges}

\subsection{Alignment and orientation}

The first experimental prerequisite is to control the EWP re-collision angle $\theta$ in the molecular frame. This implies that the molecules have to be held in a certain orientation 
in the laboratory frame, where the EWP re-collision direction $\hat{k}$ is fixed by $\hat{\epsilon}_{I R}$ (note that small deviations from that exist, which may induce some distortions to the expected dipole response [46]). Symmetry in some cases, e.g., in homonuclear diatomics, makes it sufficient to only fix the alignment of some particular molecular axis $(\uparrow)$, whereas in general, one has in addition to control the up and down directions of this axis ( $\uparrow$ ), i.e. to orient the molecule. Achieving this prerequisite is a formidable experimental challenge and it remains an active area of research [47-50]. On the one hand, HHG requires relatively high $\left(\sim 10^{17} \mathrm{~cm}^{-3}\right)$ medium densities, while the ones in which highly efficient alignment and orientation are typically demonstrated are orders of magnitude lower. This is because very high degrees of alignment and orientation demand very low initial rotational temperatures, that are attained only in very cold gas jets produced by supersonic expansion, employing (several) skimmers and possibly some means of state-selection $[51,52]$. Thus, a temperature-density trade-off has to be found. For 1D alignment, this results in rotational temperatures $\lesssim 100 \mathrm{~K}$ where significant degrees of laser-assisted alignment are already achieved at the typical HHG densities.

For non-linear molecules, a 3D alignment is required - even if one decides to restrict the probing and reconstruction to 2D. 3D alignment places higher demands on the rotational cooling, and the orientation of molecules is a yet more challenging task, since it additionally requires an asymmetric laser field that distinguishes up and down. A full control over the orientation of molecules in the laboratory frame has been demonstrated in very low density ensembles [51-53]. Extending it to media with sufficient densities for HHG is one of the chief experimental challenges we identify for progress towards the generalization of the tomographic procedure.

\subsection{Harmonic generation and characterization}

Once alignment is under control and the molecules are pinned down in the laboratory frame, an intense laser pulse (typically $I \approx 10^{14} \mathrm{~W} \mathrm{~cm}^{-2}$ ) produces HHG in the aligned medium. In the case of oriented molecules, one must ideally ensure that they are probed only from one side, i.e., the inversion symmetry of the driving laser field must be broken. This can be performed either by mixing the driving laser with its second harmonic or by using few-cycle pulses with stabilized Carrier-Envelope Phase (CEP) [54]. It turns out that gas jets required for efficient alignment/orientation (in our case: molecule density $\sim 10^{17} \mathrm{~cm}^{-3}$ and effective medium length $\lesssim 1 \mathrm{~mm}$ ), also establish medium properties that are optimal for broad-band phase matching and minimal re-absorption. By positioning the laser focus before the gas jet, phase matching is optimized for the contributions to the harmonic dipole of the shortest possible trajectories for the electron in the continuum (corresponding to excursion times $\lesssim 0.65 T_{I R}, T_{I R} \approx 2.7$ fs for an 800-nm laser) [55, 56]. The measured macroscopic harmonic signal is then essentially an amplified version of the single-molecule emission restricted to these short trajectories. Trajectory selection is very important for ensuring the validity of both the dipole factorization in Eq. 3 and the calibration procedure in Eq. 5 because, as already mentioned, different trajectories 
leading to the emission of the same harmonic photon have different ionization times and thus ionization amplitudes $\kappa$ and different continuum amplitudes $a(\vec{k})$.

For each harmonic frequency $\omega$ and orientation $\theta$, the spectral intensity, phase and polarization of the complex harmonic field $\vec{E}_{X U V}(\omega, \theta)$ are in principle required to perform the tomographic analysis. If measuring the spectral intensity of the harmonics is relatively straightforward using an XUV spectrometer, it is experimentally much more challenging to measure the XUV spectral phase and polarization.

As for the harmonic spectral phase, one has to realize first that one is dealing with a quantity $\phi(\omega, \theta)$ depending on both the harmonic frequency and the molecule orientation. Techniques such as FROG-CRAB [57] and RABBIT [11], designed for the characterization of attosecond pulses, measure the spectral phase up to a constant, i.e. up to the CEP of the attosecond pulses. This means that the phase derivative with respect to frequency, $\partial \phi / \partial \omega$, i.e. the so-called group-delay, is determined in these experiments. Repeating such measurements for several orientations of the molecule $[3,58]$ is thus not sufficient to recover the complete phase information since its variation with orientation at a given frequency remains undetected.

To access the latter, it is necessary to measure the phase derivative with respect to orientation, $\partial \phi / \partial \theta$. Doing so for several frequencies then adds redundancy to the data which can be used to cross-validate the measurements. An experimental method for such measurements is high-harmonic interferometry [33, 39, 59-61], where HHG from unaligned molecules serves as a reference, against which the phase of the aligned/oriented molecules is retrieved from the far-field interference pattern. A similar approach consists in producing HHG in mixed gases [62-64], where a different species not prone to orientation - often a noble gas - provides a phase reference for extracting the orientation-dependent phase from the interferences taking place in the mixed medium. The measurement of the phase derivatives with respect to frequency and orientation finally determines the spectral phase as a function of orientation up to a global constant, which will turn out to have no importance for orbital imaging, as wave functions themselves are defined up to an arbitrary global phase.

Eventually, the characterization of the harmonic emission can be completed by measuring its polarization state using, e.g., the reflection off a bare silver-mirror as a broad-band polarizer in the XUV [34, 65-67]. Ideally, the harmonic intensity and phase - both as functions of frequency and orientation - are measured in a polarization resolved way (as done in ref. [3]), i.e. by selecting only one polarization component of the high-harmonic radiation. Then, the measurement of the harmonic ellipticity and angle of the ellipse main axis provides both amplitude and phase of the perpendicular polarization component, resulting in a complete characterization of the high harmonic radiation.

All the techniques mentioned above are aimed at characterizing the macroscopic harmonic field $E_{X U V}(\omega, \theta)$. Since the angular distribution of the molecular medium has a finite width, the detected harmonic signal is the coherent addition of the fields emitted by molecules with various alignment angles around the mean value $\theta$ (with typical 
deviations of $\left.\pm 10^{\circ}\right)$. Retrieving the single-orientation response from the macroscopic signal is a field of high current interest [4, 62].

\subsection{Sampling the Fourier space}

The spatial quality of the orbital reconstruction is obviously determined by the sampling in $\vec{k}$, i.e., by both the discretization and the covered range of parameters - see [25] for a detailed discussion. The finite discrete sampling combined with the PW approximation gives in general different results for the orbitals reconstructed from the $x$ and $y$ dipole matrix elements. For that reason, one performs averaged reconstructions:

$$
\tilde{\varphi_{0}}(x, y)=\frac{1}{2}\left[\tilde{\varphi}_{0}{ }^{x}(x, y)+\tilde{\varphi}_{0}{ }^{y}(x, y)\right]
$$

in order to balance the distortions induced in each of the $\tilde{\varphi}_{0}^{x}$ and $\tilde{\varphi}_{0}^{y}$ reconstructions.

The truly crucial parameter is the range and sampling of $k$ values to be covered. First, it is necessary to sample around the characteristic spatial frequencies of the orbital (see Section 5.1). Second, it is beneficial to extend the range to high $k$-values. Using a mid-IR driving laser field rather than a conventional Ti:Sapphire source allows for a finer sampling and generation of much higher harmonic orders, since the spectrum cutoff scales as $I_{L} \times \lambda_{I R}^{2}[8,9]$. The inherent reduction of harmonic signal, which scales unfavorably as $\lambda_{I R}^{-5}$ [68], may be partly compensated by improved phase-matching [6971]. Such a plateau extension will be of particular importance for a large variety of species with low binding energies or photo-excited and probed in a transition state, as they will not withstand too high laser intensities.

As for the orientation, sampling $\theta$ every $10^{\circ}$ may be enough to resolve typical oscillations and nodal structures of valence shell orbitals in many cases. Another issue for the orbital reconstruction is the need to sample the $\vec{k}$-space over $360^{\circ}$. This is not a problem for asymmetric molecules, provided that they are oriented and probed only from one side at a time, as explained above. For symmetric molecules however, the symmetry of the orbital cannot be obtained from measurements at symmetric orientations. Consider for example a diatomic molecule with its internuclear axis along $(O x)$ : a quick-and-dirty interpretation of the harmonic dipole factorization in Eq. 3 may suggest to look for phase variations when probing it from $\theta$ and from $-\theta$. In fact, the two measurements provide the same observable, irrelevant of the symmetry ( $\sigma$ or $\pi$ ) of the probed orbital. This is simply because the overall orbital phase is arbitrary, and any phase convention will be carried along by the EWP, from tunnel ionization to recombination (as illustrated e.g. in Fig. 8 of [25]), while the "naive" scheme relies on fixed and independent phases for the probe EWP and for the probed orbital. Up to now, the orbital reconstructions based on the self-probing scheme were carried out on diatomics with assumed symmetries.

In view to detect the symmetry from HHG measurements, one should rather look for patterns resulting from interferences of the structured EWP with itself - such as two-center interference minima, the position of which indicate whether the interfering 
emitting centers (here the orbital lobes) have opposite or equal signs [72, 73]. In the same spirit, it has been proposed to steer the electron trajectory in the continuum to make it recollide with the core from a different direction, and probe the orbital phase changes at the quadrant boundaries. Steering can be performed using an elliptically polarized laser field or a mixing of cross-polarized $\omega_{I R}-2 \omega_{I R}$ fields [74-76]. Recently, it was proposed that the orbital symmetry could also be inferred from polarization measurements [77].

\subsection{Experimental orbital reconstruction}

We illustrate the application of the tomographic procedure with the $\mathrm{N}_{2}$ orbital reconstructions reported in [3]. A cold gas jet with 90-K rotational temperature is produced by supersonic expansion. Nonadiabatic alignment is obtained by focusing an 800-nm 55-fs laser pulse at $5 \times 10^{13} \mathrm{~W} \mathrm{~cm}^{-2}$ in the jet, creating a rotational wavepacket that periodically rephases. At revival, a field free molecular alignment along the laser polarization is obtained with $\left\langle\cos ^{2} \theta>\approx 0.55\right.$. A second laser pulse focused at $1.2 \times 10^{14} \mathrm{~W} \mathrm{~cm}^{-2}$ generates harmonics in the aligned medium. Two grazingincidence $\left(11.5^{\circ}\right)$ reflections on a toroidal mirror and a flat mirror coated with gold preferentially transmit the s-polarized component of the harmonic beam; the generating laser is kept s-polarized while the molecular alignment axis is rotated to predominantly detect the HHG polarization component parallel to the generating laser field. Finally, a magnetic-bottle electron spectrometer is used to perform the measurement of the harmonic spectral amplitude and phase using the RABBIT technique: the two-photon $\mathrm{XUV}+\mathrm{IR}$ photoionization of a target gas is induced by the harmonic beam in presence of a third laser beam focused at $\approx 10^{11} \mathrm{~W} \mathrm{~cm}^{-2}$, the delay of which is controlled with attosecond accuracy. The measurements were performed in the spectral range H17-H31 for a sampling of $\theta$ with $10^{\circ}$-steps in the range $0-90^{\circ}$. The symmetry of the orbital was then assumed in order to cover the range $90-360^{\circ}$. Argon $\left(I_{p}^{A r}=15.76 \mathrm{eV}\right)$ provided the reference gas $\left(I_{p}^{N_{2}}=15.58 \mathrm{eV}\right)$.

Figure $2 \mathrm{~b}$ ) displays the experimental reconstruction. It is to be compared to the Hartree-Fock Highest Occupied Molecular Orbital (HOMO) projected onto the (xOy) plane (Fig. 2a) and to a simulated reconstruction (Fig. 2c). The latter was obtained with PW dipoles filtered for the experimental harmonic (H17-31) and orientation (0$\left.90^{\circ}\right)$ samplings, and projected along the laser polarization direction. The experimental reconstruction is remarkably similar to the simulated one. The restricted harmonic span results in a filtering of the spatial frequencies of the orbital: consequently the reconstructed orbital is elongated with extra oscillations due to the discrete sampling. However it retains the main features of the Hartree-Fock orbital and exhibits the characteristic three-lobe shape with alternating signs and nodal surfaces passing through the nuclei.

The experimental reconstruction presented in Fig. 2b is significantly different from the one published in [2]. The main reason is that in this seminal work, only the amplitude of the harmonic emission was measured and both the phase and the polarization were 


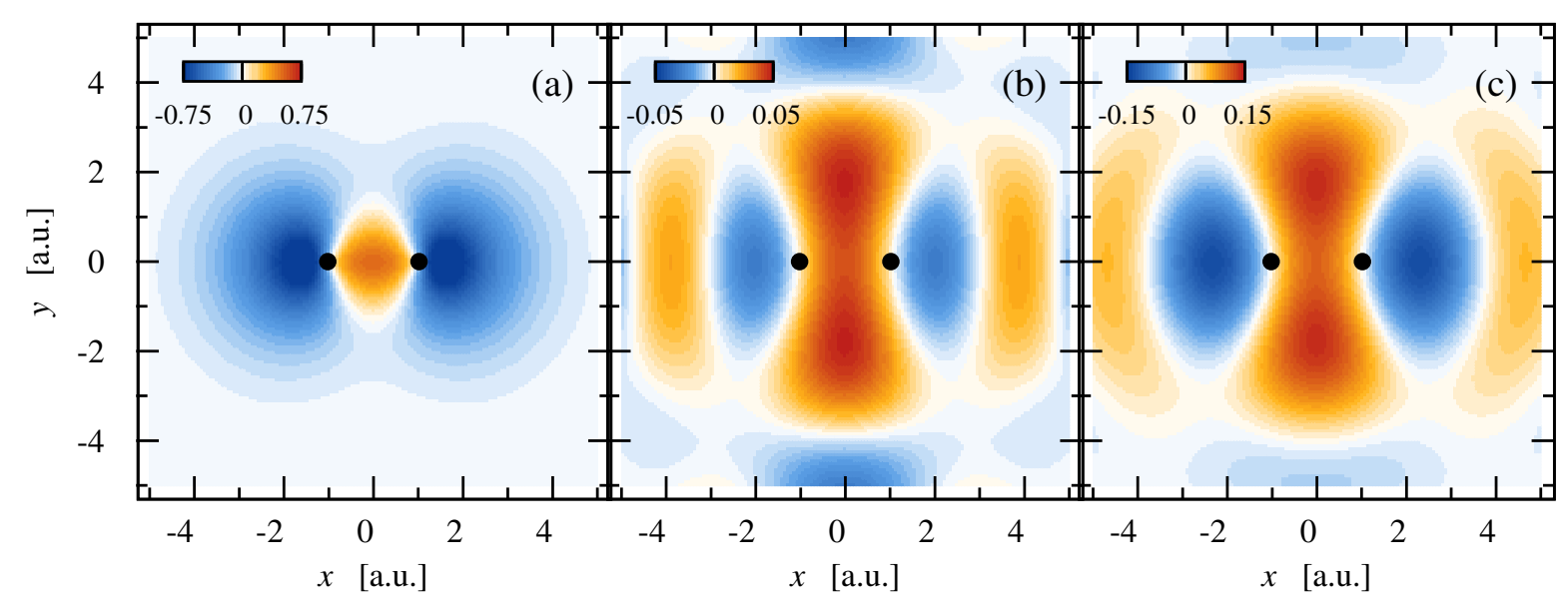

Figure 2. Highest Occupied Molecular Orbital of $\mathrm{N}_{2}$ : (a) Hartree-Fock orbital; (b) reconstruction from experimental data; (c) reconstruction from theoretical PW dipoles filtered as in the experimental conditions. Black dots indicate the nuclei positions.

assumed. In particular, a $\pi$ phase jump at harmonic 25 was imposed at all probing angles, which was not observed in the conditions of Ref. [3]. Another difference lies in the sampling: in [2], the harmonic range was H17-43. A difference in sampling may also be the reason for the discrepancy with the theoretical reconstructions performed for $\mathrm{N}_{2}$ in $[78,79]$ (see Section 5.1).

\section{Access to the recombination dipole moment: Open questions}

\subsection{Calibration for the continuum amplitude $a(\vec{k})$}

The calibration of the harmonic signal with a reference system in Eq. 5 has been shown to be quite efficient in cancelling the complex amplitude $a(\vec{k})$ accumulated by the EWP in the continuum (see Eq. 3) [3, 58]. In particular, its phase amounts to several tens of $\pi$ radians and overshadows the subtle phase variations of the recombination dipole moment if not properly removed from measured data. Note that the calibration also presents the advantage of compensating for the spectral response of the detection system that otherwise should be determined accurately. However, the availability of a reference gas of same ionization potential is quite limited. Besides, when several orbitals with significantly different $I_{p}$ 's participate simultaneously in the emission process (see Section 5.2 ), the calibration is efficient only for one contribution (in general the HOMO), and leaves part of the continuum phase for the other ones. Thus, a theoretical issue remains, regarding the possibility to produce reliable estimates of the time evolution of the EWP in these molecular systems. Under the assumption of purely laser-driven continuum dynamics, knowledge of $I_{p}$ and of the laser parameters with sufficient precision should allow using theoretical predictions. Let us mention that if the response of the reference system does not vary smoothly in the considered spectral domain, for instance because of a Cooper minimum or a resonance, a precise calculation of the recombination dipole, 
most likely beyond the PW approximation, must be used to compensate for this effect in Eq. 5 - bringing considerable complications to the data analysis [80, 81]. One can anticipate that this latter point will be of importance when wider spectral ranges are considered, notably in relation with the advent of mid-IR laser sources. Note finally that the calibration does not remove the angle-dependent quantum diffusion in the continuum.

\subsection{Calibration for the ionization amplitude}

The operational expression of the dipole in Eq. (5) contains the $\theta$-dependence of the tunnel ionization amplitude of the molecule. Theoretically, it is difficult to describe accurately this dependence because there is no easy way to extend the atomic ADK approach [82] to the case of a non-spherically symmetric system. Indeed, to compute tunnel ionization amplitudes, one needs an accurate representation of the asymptotic form of the molecular wave functions, not available from standard quantum chemistry codes. Various schemes have been explored to improve this asymptotic description $[83,84]$. Alternatively, solving the TDSE for the molecule in the strong IR field provides computed yields containing all the entangled contributions to ionization, including multiphoton and stepwise processes, in addition to tunnelling which is the only one we are interested in for HHG.

\subsection{What dipole is it?}

The detailed balance principle states that the recombination dipole is merely the complex conjugate of the (single-photon) photo-ionization (PI) dipole, since PI is the reverse process of radiative recombination. A nice demonstration of the direct relationship existing between HHG and PI spectra, is provided by the signature of the presence of a Cooper minimum in the harmonic spectrum of Argon, at a frequency very close to the one in the photoelectron spectrum [80, 81]. The "giant" resonance in photoelectron spectra in xenon has also been recently observed in HHG spectra [85]. However, several questions remain open, when trying to establish a direct correspondence between the two processes. This is particularly true in molecular systems where the precise calculation of the angle-dependent PI dipoles, including the correct phase-shifts (or scattering-phase) remains a challenge for theory [79, 86, 87].

Three important questions are currently discussed:

- In principle, the detailed balanced principle does not strictly apply to HHG since the driving laser field is present during the recombination process and may induce a dressing of the molecular states (polarization of the molecule). For harmonic orders close to the cutoff region, where the laser field at recombination is close to zero, such an effect is expected to be small but it may influence harmonic orders well in the plateau region. Single-active-electron TDSE simulations have shown, both in atoms [87] and in $\mathrm{H}_{2}^{+}[44,88]$, that this effect is negligible and that field-free recombination dipoles may indeed be extracted from the HHG signal. 
- What happens when sharp - long lived - resonances are present in the PI dipole? Do they appear in the HHG-extracted recombination dipole despite the attosecond dynamics of the emission process? Indeed, HHG is a coherent process and, through phase matching, one may select the contributions to the EWP with the shortest excursion times $\lesssim 0.65 T_{I R} \approx 1.7 \mathrm{fs}$ for an 800-nm laser. It is questionable whether this leaves enough time for building sharp resonances the timescales of which would be larger than, say, 10 fs. If not, the HHG dipole may be "cleaned" from such resonances, as a result of this "temporal filtering". This question has emerged recently in the community and is currently much debated [89].

- Finally, it is clear that the HHG-extracted recombination dipole deviates from the PI dipole when many different orbitals contribute coherently to the harmonic emission. Disentangling their contributions to HHG measurement will be in general problematic, while in PI, the energy of the ejected electron is a signature of the ionized orbital (see Section 5.2).

Note that HHG measurements give in principle access to both the modulus and the phase of the recombination dipole vector - which allows performing the inverse Fourier transform and ultimately gives access to the orbital, in contrast to PI experiments where the spectral phase is lost. Obviously, the phase is a crucial element in this orbital reconstruction scheme since it encodes the structure of the orbital through changes of sign (or $\pi$ phase jumps) at the characteristic spatial frequencies.

\section{Analysis of the recombination dipole: Accessing the molecular orbital(s)}

In this section, we review three major issues that must be carefully considered prior to applying the apparently straightforward tomographic analysis to any experimental molecular HHG data: deviations from the PW approximation, participation of multiple ionization channels, and correlated multi electron processes. We illustrate our purpose with simulations for the nitrogen molecule $\mathrm{N}_{2}$ which come as a complement to the analysis presented in [3].

\subsection{Validity of the plane-wave approximation}

The plane-wave approximation for the continuum excursion is by far the most criticized pillar of the SFA, when applied to molecules as much as in atoms. Indeed, this approximation (equivalent to using the 1st-Born approximation in collision theory), is a priori inadequate in the range of relatively low electron kinetic energies relevant to HHG; in particular, it does not contain the scattering phase-shifts characteristic of the system. This has been one of the main objections to the tomographic procedure and the subject of theoretical investigations. Numerical experiments were performed to obtain the harmonic dipole moment from the solution of the (single-active-electron) TDSE in model systems. Application of the (PW) tomographic procedure resulted in very 

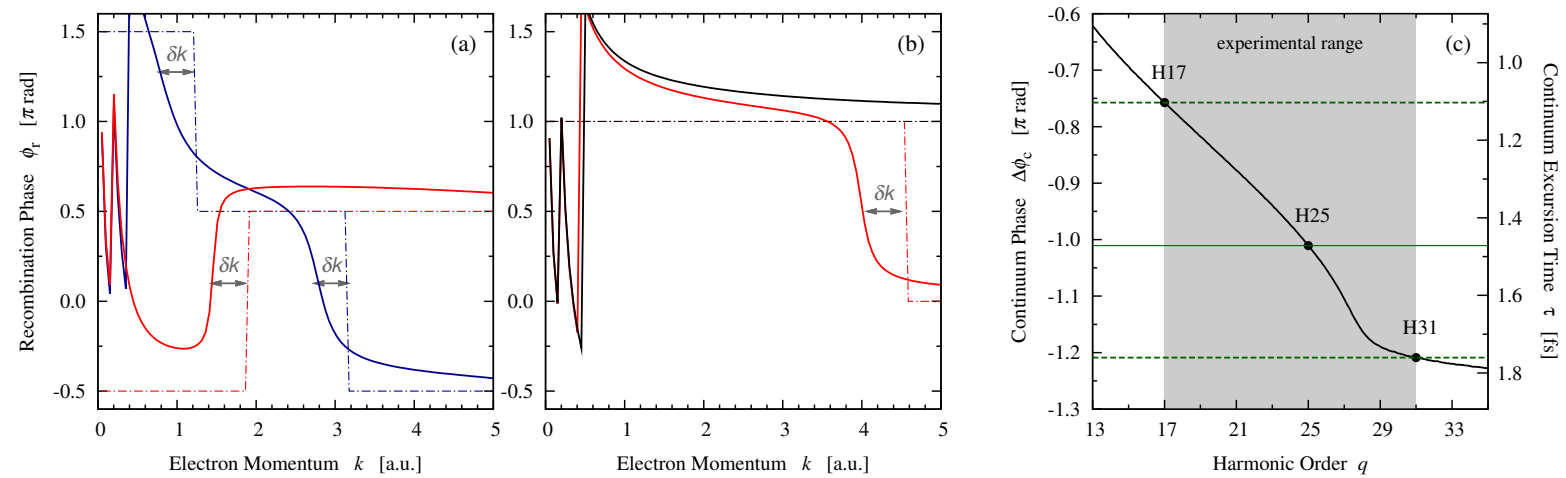

Figure 3. Phase of the recombination dipole matrix elements parallel to the laser polarization for the $\mathrm{N}_{2} \mathrm{HOMO}$ (a) and HOMO-1 (b) as a function of the asymptotic electron momentum $k$. PW/CW dipole phases are shown in dashed/solid lines for $\theta=0$ (blue), 45 (red) and 90 (black) degree. Calculations for HOMO-1 are performed using the velocity form. The continuum phase difference $\Delta \phi_{c}$ is shown in (c) as a function of the harmonic order. It was computed with SFA for an 800-nm laser field with intensity $1.2 \times 10^{14} \mathrm{~W} \mathrm{~cm}^{-2}$. and the binding energies for the HOMO $(-15.58 \mathrm{eV})$ and HOMO-1 $(-17.00 \mathrm{eV})$. The horizontal lines indicate the phase differences and excursion times for the first (H17), middle (H25) and last (H31) harmonics of the considered spectral range.

good orbital reconstructions in the three studied cases (symmetric, antisymmetric and asymmetric orbitals) [54]. On the other hand, application of the procedure to accurate photoionization dipoles calculated using quantum chemistry codes led to distortions of the reconstructed orbitals [79].

In order to better evaluate the influence of the ionic potential, we performed a case study using analytic Coulomb waves $(\mathrm{CW})$ for the scattering states and Hartree-Fock orbitals of the nitrogen molecule for the initial state: either the HOMO or the orbital lying just below (HOMO-1). Both ionization channels have been shown to participate simultaneously in HHG from $\mathrm{N}_{2}[3,34,90]$, as will be discussed in Section 5.2. In a first step, we here consider their contributions independently. Using $\mathrm{CW}$ can be seen as a first order improvement of the PW description, since asymptotically, the $\mathrm{N}_{2}^{+}$ion acts on the recolliding electron just like a proton.

Figure 3 compares the phase of the recombination dipoles $\vec{d}(\vec{k})$ computed with the PW and CW approximations for three different angles, $\theta$, of $\vec{k}$ relative to the molecular axis. The HOMO (HOMO-1) PW dipole has a phase that is a multiple of $\pi / 2(\pi)$ for all $k$, i.e. it is purely imaginary- (real-) valued. This is a direct consequence of the symmetry properties of the orbitals, dipole operator and Fourier transform. Sharp $\pi$-rad phase jumps indicate sign changes that are a direct manifestation of the spatial structure of the orbital at characteristic values of $k$. These characteristic spatial frequencies depend on the recollision angle, which leads to a shift of the phase jumps location with increasing angle [72, 91]. Switching to Coulomb waves corresponding to an effective ion charge of $Z=1$ provides strongly oscillating phases for $k \lesssim 0.8 \mathrm{a} . \mathrm{u}$. (i.e. kinetic energies $\lesssim 9 \mathrm{eV}$ ). The very rapid variations at these low momenta is a direct imprint left by the $\mathrm{CW}$, as 
can be seen from their partial wave expansion [92], where each angular momentum $\ell$ contributes with a phase $\arg [\Gamma(\ell+1+\mathrm{i} Z / k)]$. However, at higher momenta - including values typical for HHG - both series of curves show similar patterns, with the following differences: i) beside being smoothed, the $\pi$ jumps are translated towards lower $k$ values by an amount $\delta k \approx 0.4$ a.u., and ii) the phases are vertically shifted by a global term $\delta \phi_{r}$ that decays smoothly as $k$ increases.

The observed momentum translation of the phase features in i) translates to an energy shift of $\approx 15 \mathrm{eV}$ in the spectral region of $(0.9$ a.u. $\lesssim k \lesssim 2$ a.u. $)$ relevant to most HHG experiments using $800-\mathrm{nm}$ driving lasers. This corresponds to the fact that the recolliding electron, as it approaches the core, experiences an additional acceleration by the ionic potential, with typical energy gain of $\approx I_{p}[93]$. This is why in the interpretation of self-probing experiments, as a first correction for the errors introduced by the PW approximation, one modifies the relation between the recolliding electron wave number $k$ at recollision and the measured XUV photon energy $\omega$ by the heuristic relation: $\hbar \omega=\hbar^{2} k^{2} / 2 m$. A similar conclusion was reached using two-center Coulomb waves in the case of $\mathrm{H}_{2}^{+}$[94]. Consequently, the heuristic relation was used for the reconstructions in Fig. 2: the harmonic range H17-31 then corresponds to 1.39 a.u. $<k<1.88$ a.u., and thus covers the crucial zone where the dipole phase changes rapidly, i.e., close to a characteristic spatial frequency of the HOMO. While the (translated) phase jumps are imprints of the bound orbital structure, the global phase shift $\delta \phi_{r}$ mentioned in ii) corresponds to the $\mathrm{CW}$ scattering phase. In the experimental harmonic range, an average value of $\delta \phi_{r} \approx \pi / 4$ rad is obtained for both HOMO and HOMO-1 dipole phase. This means that a $-\pi / 4$ rad rotation of the CW dipoles results in mostly imaginary/realvalued dipoles for the HOMO/HOMO-1, similarly to the PW dipoles.

In Fig. 4, we display tomographic reconstructions (frames e-h) using the CW dipoles in the same conditions as in Fig. 2. Here, we used the asymptotic $k$-values given by the usual relation (Eq. 4), corresponding to 0.88 a.u. $<k<1.54$ a.u. This allows probing the same "slice" of the dipole (around the characteristic $\pi$-phase jump). To account for the PW tomographic procedure, the different $k$-values are then shifted by $\delta k$ in the reconstruction. Due to the smoothing of the phase jumps as well as to the phase variation within the relevant $k$-range, the CW dipoles for HOMO / HOMO-1 are not purely imaginary / real valued, even after the $-\pi / 4$ phase correction mentioned above. Using these corrected dipoles (with adapted symmetries) results in complex reconstructed orbitals. However, the real part of the reconstructed HOMO/HOMO1 (shown in Figs. 4e and $\mathrm{f}$ respectively) are approximately 10 times larger than the corresponding imaginary parts (Figs. $4 \mathrm{~g}$ and $\mathrm{h}$ ). It is noticeable that the shapes of the orbitals thus obtained are very similar to the ones reconstructed from PW dipoles (Figs. $4 c$ and $d)$.

We thus conclude that, in this simple case, correcting for i) the momentum translation and ii) the global phase shift allows relatively accurate orbital reconstructions the precision of which is mainly limited by the accessible harmonic spectral range. 


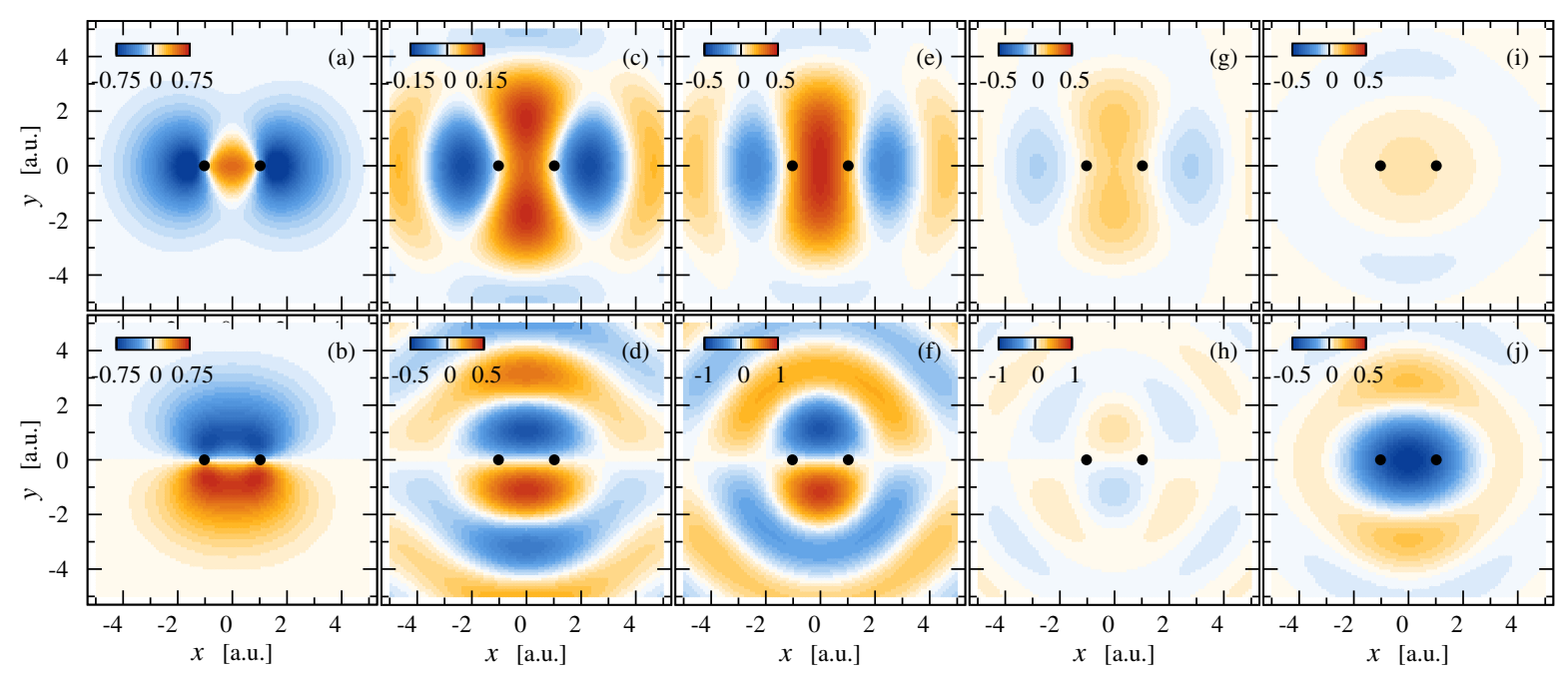

Figure 4. HOMO and HOMO-1 of $\mathrm{N}_{2}$ and contributions to their tomographic reconstructions in the experimental spectral range (harmonics 17-31). Hartree-Fock HOMO (a) and HOMO-1 (b). Orbitals reconstructed from the PW dipoles of the HOMO (c) and HOMO-1 (d). Real/Imaginary part of the orbitals reconstructed from the Coulomb-wave dipoles of the HOMO (e)/(g) and HOMO-1 (f)/(h) with a $\pi / 4$ global phase shift applied. Real (i)/Imaginary (j) part of the orbital reconstructed from the Coulomb-wave HOMO-1 dipole, multiplied by the continuum phase factor $\exp \left(i \Delta \phi_{c}\right)$ (the HOMO symmetry is enforced in the reconstruction). The deviation from $-\pi$ of $\Delta \phi_{c}$ results in a small contribution to the real part of the orbital. Frames (a) and (c) correspond to the frames (a) and (c) of Fig. 2.

Note that beside the basic corrections i) and ii) to the plane-wave approximation discussed above, there exist other possibilities of improvements. For instance, by writing the tomographic procedure using the velocity form of the recombination dipole, one can include in a first-order perturbative approach the deviation from the plane wave due to the ion field [4]. Finally, indirect retrieval procedures using iterative algorithms have also been proposed to recover the orbital without the plane-wave approximation [78, 95].

\subsection{Contribution of several channels}

An additional difficulty comes into play in molecules that is absent in atoms: the possibility to tunnel ionize through several channels, namely from the HOMO as well as from the closest lying valence orbitals (HOMO-1, HOMO-2 ...), as already demonstrated in $\mathrm{N}_{2}[3,34,90,96], \mathrm{CO}_{2}[33,97,98]$, and $\mathrm{H}_{2} \mathrm{O}$ [99]. Indeed, the probability to tunnel ionize decays exponentially with the binding energy. While in atoms, subshells are typically separated by tens or hundreds of $\mathrm{eV}$, valence orbitals in molecules are only a few $\mathrm{eV}$ apart and may be simultaneously tunnel ionized by the strong laser field. These different ionization channels provide different coherent contributions to the harmonic emission at recollision.

This raises the following question: Is it possible to disentangle their respective contributions in view to reconstruct each participating orbital? First, this requires 
the absence of coupling between the different channels, couplings that may be induced by the laser field and/or the electron correlation [34]. Second, one must consider the recombination dipoles corresponding to each channel. In the particular case of $\mathrm{N}_{2}$, only the first two highest occupied orbitals - separated by $\approx$ an IR photon energy $(1.55 \mathrm{eV})$ - are expected to contribute. We have seen above that the HOMO (HOMO-1) PW dipole is purely imaginary (real) valued due to the orbital different symmetries. This is still approximately the case for the CW dipoles provided that a $-\pi / 4$ global phase shift correction is applied, so that the reconstructed HOMO and HOMO-1 in Fig. 4ad) present very small imaginary parts that would represent "leakage" of information in the other quadrature and mixing of the two contributions. Could we say that the two contributions are also disentangled in the real and imaginary parts of the calibrated harmonic dipole? In fact, the continuum dynamics in the two channels is different due to the different ionization energies: the calibration of the harmonic dipole by the reference (argon) gas removes the continuum amplitude $a(\omega)$ for the HOMO contribution (see Eq. 3) but leaves in particular the continuum phase difference for HOMO and HOMO-1: $\Delta \phi_{c}(\omega) \approx \Delta I_{p} \tau$, with $\tau$, the mean excursion time in the continuum of the electron trajectory corresponding to the considered harmonic frequency $\omega$ [100]. This is shown in Fig. 3c). At this laser intensity, $\Delta \phi_{c}$ varies around $-\pi$ by $\pm 0.2 \pi$ in the H17-31 spectral range. This deviation from $-\pi$ mixes somewhat the two orbital contributions. To illustrate this, we show in Fig. 4e,f) the real/imaginary parts of the orbital reconstructed from the CW-HOMO-1 dipole multiplied by the continuum phase factor $\exp \left(i \Delta \phi_{c}\right)$ with enforced HOMO symmetry. It comes as no surprise that the HOMO-1 contribution now goes mostly to the imaginary part with a factor 10 compared to the real part; the latter thus hardly affects the real HOMO reconstruction when adding up the two contributions.

In the experimental reconstructions, we thus used the imaginary/real part of the measured dipole to recover the HOMO/HOMO-1 (enforcing the corresponding symmetry). An image of the HOMO-1 reconstruction can be found in [3].

In summary, for the limited spectral range considered here, the contributions from the HOMO and HOMO-1 to the emission of $\mathrm{N}_{2}$ can be practically disentangled (or disentanglable) in the imaginary and real parts of the harmonic dipole respectively. This allows reconstructing both orbitals without significant distortions that would be due to the mixing of the different channel contributions to the HHG spectra.

We note however that it will not always be straightforward - or even possible - to meet proper conditions to disentangle multi-orbital contributions. For example, in $\mathrm{CO}_{2}$ probed at angle $\theta$ close to $0^{\circ}$, a $\pi$ phase difference between the HOMO and HOMO-2 contributions was identified as responsible for destructive interferences, manifesting as a minimum in the harmonic spectrum. The signature of such a so-called "dynamical" minimum is that its spectral position varies with the laser parameters (intensity and wavelength), in contrast with "structural" minima associated to characteristic spatial frequencies [58, 60, 62, 98, 101-103]. While it prevents a priori from performing a clean tomographic analysis of the harmonic signal, the dynamical minimum in $\mathrm{CO}_{2}$ was exploited in $[33,97]$ to retrieve fundamental informations on the ion dynamics during 
the generating process. Moreover, Vozzi et al. found conditions where the dynamical minimum disappears, using few-cycle mid-IR $(\lambda \approx 1.5 \mu \mathrm{m})$ laser pulses, and thus could successfully reconstruct the $\mathrm{HOMO}$ of $\mathrm{CO}_{2}$ [4]. Isolating the $\mathrm{HOMO}$ contribution by adjusting the generating conditions was also recently demonstrated in $\mathrm{N}_{2}$ [104].

\subsection{Correlated processes}

Once it is realized that multichannel dynamics occur in molecules, the next step is to consider correlated multi-electron processes. This crucial point directly challenges the validity of the single-active electron approximation, adopted by the vast majority of models in strong-field physics. Correlated dynamics are behind a rich variety of different phenomena arising essentially from two simple facts:

(i) The laser field acts on all electrons of the molecule, i.e. also on those that remain bound. It can thus polarize both the bound wavefunction in the neutral molecule as well as the molecular ion [105]; and it may excite the molecular ion on a sub-cycle time scale, changing the electronic configuration of the ion between ionization and recombination in HHG, thus resulting in channel coupling and the appearance of cross-channels [34]. The larger the molecules, the stronger the bound electrons are likely to be affected by the laser field.

(ii) The electrons of the molecule, including the one being ionized, interact among each other through Coulomb repulsion. As an electron is being tunnel ionized, it interacts with the remaining electrons and may thereby electronically excite the ion $[106,107]$. When the continuum electron returns, it will again interact with the bound electrons in the ion, possibly exciting it $[89,108]$.

These few examples at least demonstrate that expressing a true multi electron dynamics in terms of single electron events quickly becomes awkward, and one loses the intuitive and elegant interpretation of HHG provided by the semiclassical model.

Concerning ab initio simulations, since the complexity of the TDSE increases exponentially with the dimensionality of the problem, brute force calculations are still limited to a maximum of 2 active electrons [109]. Of the methods able to deal with more electrons, multi-configuration time-dependent Hartree-Fock (MCTDHF) may be the most promising one, as it fully includes correlation but also allows to gradually "switch" it on and off by including more or less configurations $[105,110]$.

To date, strong-field theorists are just starting to gain qualitative and quantitative understanding of multi-electron phenomena. A first step that brings valuable insight is made by reformulating the matrix elements of the SFA three-step model for multielectron systems $[95,111,112]$. The recombination dipole matrix element can then be written as:

$$
\vec{d}(\vec{k})=\left\langle\varphi^{\mathrm{d}}|\hat{\mathbf{r}}| \varphi_{\vec{k}}\right\rangle+\vec{d}^{\mathrm{ex}} .
$$

Here, $\varphi^{\mathrm{d}}$ is the Dyson-orbital, i.e. the scalar product of the $f$-electron wavefunction of the neutral and the $(f-1)$-electron wavefunction of the ionic core, where integration 
runs over the first $(f-1)$ electron coordinates. This orbital takes the role of the initialstate orbital $\varphi_{0}$ in the single-active-electron formulation and can be interpreted as an electron-hole in the ion [3,33]. For an alternative, more general, definition of the hole (density), see $[113,114]$. The "exchange correction" term $\overrightarrow{d^{e x}}$ contains contributions by all other bound orbitals of the neutral, but becomes important only if orbital relaxation upon ionization is significant [25]. In $\mathrm{N}_{2}$, such relaxation is negligible [89], allowing a direct recovery of the Dyson orbital. As theoretical understanding of these effects will improve in the future, it is likely that self-probing experiments can be used as sensitive probes for correlated multi-electron dynamics.

\section{Access to the dynamics of the radiating system}

We now consider both the temporal and spatial properties combined in HHG and raise the ultimate (for the present concern) question: Can we image a dynamics occurring in the radiating system?

\subsection{Imaging the hole left by tunnel ionization}

The dynamics of a "hole" - that is, the space left vacant by an absent electron created in an atom or a molecule by single-photon ionization can be extremely fast, with the early response lasting about 50 as [114]. In a polyatomic molecule, electron correlation can drive the hole across a few Angströms in just a couple of femtoseconds [115]. Resolving this motion in space and time is an enticing prospect and a formidable challenge [116]. Simultaneous tunnel ionization from two orbitals creates a coherent wavepacket (a "hole") in the molecule that evolves during the EWP excursion, and that is probed at the recombination instant. In the case where the orbital contributions can be disentangled, the coherent superposition of the orbital reconstructions can be interpreted as a snapshot of the hole at recombination [3]. In multielectron language, the Dyson wave-packet extracted via time-resolved HHG-tomography (interpreted as the hole left in the ion) is a coherent superposition of the Dyson orbitals associated with all channels contributing to the signal (provided that the EWP freed in each channel can be factorized, i.e. is about the same [25]). Varying the generation parameters (laser intensity and wavelength, long trajectories) provides different ways for controlling the EWP recombination time and thus for probing the hole at different instants.

A currently debated question is the value of the initial phase between the different channels because it determines the initial location and shape of the hole after ionization $[33,97]$. Such a phase could appear between the different ionic states due to nonadiabatic dynamics of electron rearrangement during tunnel ionization. While it does not seem to be the case in $\mathrm{CO}_{2}$, recent experiments give indication for a significant phase in $\mathrm{N}_{2}$ for cross-channels [34].

Can the above study be generalized to a more conventional pump-probe scheme

to study dynamics not induced by the HHG driving laser field? For instance, to 
follow the "frontier" orbital of a photo-excited system? A number of new issues arise. Experimentally, in dynamical studies of photo-excited systems, the HHG emission by the pumped molecules will represent only a small fraction of the total signal since the pumping efficiency is presumably below $10 \%$. It may thus be necessary to implement advanced detection techniques aiming at increasing the signal-to-noise ratio, such as excitation gratings $[28,39]$.

\subsection{Compromise temporal/spatial resolution}

A more fundamental problem is the trade-off between temporal and spatial resolutions that must be performed because the different harmonic orders are associated with different electron trajectories and thus with different recollision times. A broader HHG plateau provides a wider Fourier domain - thus a finer spatial resolution, but it results from events occurring over a larger time window, which spoils the temporal resolution. Consequently the single image that they provide altogether may be blurred or even distorted if the studied dynamics is too fast [116]. If one leaves aside the encoded spatial information, the optimal temporal resolution is obtained by considering the ultrashort recollision delays between two consecutive odd harmonic orders i.e. the group delay dispersion related to the so-called attochirp [117]. At laser intensities $\approx 10^{14} \mathrm{~W} \mathrm{~cm}^{-2}$, the group delay dispersion is of the order of $100 \mathrm{as} / 2 \omega_{0} \approx 20,000 \mathrm{as}^{2}$ for a $800 \mathrm{~nm}$ laser. This indeed provides an extreme temporal resolution that can be extracted from the experimental data and combined with a priori knowledge of the spatial structure of the orbitals to reconstruct the dynamics occurring in the molecule, as performed in [33, 97].

In the tomographic procedure, a fair resolution trade-off can be reached by using only a group of harmonic orders (e.g. H17-31 generated in $\mathrm{N}_{2}$ ) to reach an Ångstrom spatial resolution, but limiting the temporal resolution to $\approx 600$ as [3]. The latter may be sufficient to probe a molecular electron hole wave-packet as it stands on one side of the molecule - since its dynamics has a fs period ( $3 \mathrm{fs}$ in the case of the HOMO/HOMO1 superposition in $\mathrm{N}_{2}$ ) and the space resolution is enough to distinguish lobes and nodal surfaces, such as the three lobe structure of the nitrogen HOMO.

Note that the reconstructed images are distorted - not just blurred - by the difference in continuum phase accumulated by each channel: as discussed above, the continuum phase $\Delta \phi_{c}$ somewhat mixes the orbital contributions but the corresponding distortions remain small in our case. Furthermore, the generation parameters such as laser intensity and wavelength can be used to control $\Delta \phi_{c}$, and in particular to decrease its variation in the measured spectral range and minimize the corresponding distortion. In conclusion, one can state that a reasonable trade-off can be reached, making it possible to obtain both Ångström and sub-femtosecond resolutions. 


\section{Conclusion}

We have reviewed the experimental and theoretical challenges raised by the tomographic imaging of valence orbitals using the "self-probing" scheme. Experimentally, it relies on the full characterization of the attosecond emission from aligned/oriented molecules interacting with a strong laser field. Theoretically, it recovers from this data the amplitude and phase of the molecular-frame recombination dipole that gives direct access to the bound orbital through a Fourier transform. This procedure involves a number of approximations/simplifications that need to be assessed carefully. In particular, we have shown that the spectral phase of the Coulomb-wave recombination dipole encodes the orbital structure, that can be recovered using first-order corrections to the plane-wave approach. Moreover, one can find conditions where multi-channel contributions are disentangled in the real and imaginary parts of the harmonic dipole. Finally, a compromise between temporal and spatial resolutions must be performed but a reasonable trade-off can be reached, making it possible to obtain "snapshots" of electronic wavepackets with both Ångström and sub-femtosecond resolutions.

How much can these conclusions be generalized? It is far beyond the current knowledge to answer this question. Presumably, this will not apply to a number of systems, due to multi-electron effects and structured continua. However, in some cases, one may be helped by the "cleaning" of the continuum from sharp resonances performed by the attosecond timescale of the emission process (see Section 4.3). Another important question is the feasibility of time-resolved orbital tomography, i.e. of making a "movie" of the changes in the valence orbitals, e.g., in the course of a chemical change. This would give direct access to the electron cloud dynamics as well as to structural information such as bond lengths and molecular geometry. A difficulty is that both types of information are inherently intertwined via the "vibronic" structure of the excited molecular states. This has been demonstrated experimentally in relatively simple systems such as $\mathrm{H}_{2}$ and its isotopes [29, 118], $\mathrm{N}_{2}$ [104] or even $\mathrm{H}_{2} \mathrm{O}$ [99], and theoretically for a collection of larger molecules [119-121].

In spite of these difficulties, these benchmark studies clearly evidence the richness of information encoded in the measurable complex HHG dipole. The tomographic procedure is nothing but a way of retrieving and presenting the information, and alternative methods - at first glance less spectacular but in principle as informative as orbital imaging - can be invoked when tomography is not applicable. Several significant advances in this direction have already been achieved in unaligned molecules using highharmonic spectroscopy. They include:

(i) Following in time the ultrafast dissociation of $\mathrm{Br}_{2}$ molecules [39]; The principle of the technique is to monitor the harmonic signal emitted by molecules brought in a superposition of the fundamental and of a dissociative state with the help of a pump pulse. Depending on the internuclear distance, destructive or constructive interferences can take place between the amplitudes originating from each states, and one can follow the transition between harmonic emission from molecular $\mathrm{Br}_{2}$ 
to the limiting case of separated $\mathrm{Br}$ atoms (at distances $>9 \AA$ ).

(ii) Exploring conical intersection dynamics in the $\mathrm{NO}_{2}$ molecule [122]. The dependence of the harmonic signal on the excitation time delay contains the signature of the nuclear motion involving the simultaneous bending and asymmetric stretching of the $\mathrm{O}-\mathrm{N}-\mathrm{O}$ bonds, close to the crossing of a conical intersection. This provides original information about the evolution of the electronic and nuclear molecular wave-packets in the course of the dissociation $\mathrm{NO}_{2} \rightarrow \mathrm{NO}+\mathrm{O}$.

In conclusion, high harmonic generation appears as a most promising tool for the development of attochemistry, i.e. the perspective of following chemical reactions by probing and ultimately steering electrons and nuclei on the attosecond time-scale. From the experimental point of view, accessing the complete recombination dipole requires to fully characterize the harmonic dipole vector in the molecular frame: besides the improvement of light characterization methods in the XUV domain, it implies the development of efficient alignment/orientation methods of larger molecules, ideally in three-dimensions. From the theoretical point of view, the effort will be concentrated on the interpretation of the complex dipole. Tomography provides one way of extracting and representing the attosecond and Angström resolved information, but it is certainly not the only one and alternative efficient methods emerge as HHG-based attochemistry grows. The dream of observing the electronic and structural changes inside a molecule during a chemical reaction becomes reality!

\section{References}

[1] Krausz F and Ivanov M Y 2009 Rev. Mod. Phys. 81 163-234

[2] Itatani J, Levesque J, Zeidler D, Niikura H, Pepin H, Kieffer J C, Corkum P B and Villeneuve D M 2004 Nature 432 867-871

[3] Haessler S, Caillat J, Boutu W, Giovanetti-Teixeira C, Ruchon T, Auguste T, Diveki Z, Breger P, Maquet A, Carre B, Taïeb R and Salieres P 2010 Nature Physics 6 200-206

[4] Vozzi C, Negro M, Calegari F, Sansone G, Nisoli M, De Silvestri S and Stagira S 2011 Nature Physics 7822

[5] Altucci C, Velotta R and Marangos J P 2010 J. Mod. Opt. 57 916-952

[6] McPherson A, Gibson G, Jara H, Johann U, Luk T S, Mcintyre I A, Boyer K and Rhodes C K 1987 J. Opt. Soc. Am. B 4 595-601

[7] Ferray M, L'Huillier A, Li X, Lompre L, Mainfray G and Manus C 1988 J. Phys. B 21 L31-L35

[8] Corkum P B 1993 Phys. Rev. Lett. 71 1994-1997

[9] Schafer K J, Yang B, Dimauro L F and Kulander K C 1993 Phys. Rev. Lett. 70 1599-1602 
[10] Lewenstein M, Balcou P, Ivanov M Y, L'Huillier A and Corkum P B 1994 Phys. Rev. A 49 2117-2132

[11] Paul P M, Toma E S, Breger P, Mullot G, Auge F, Balcou P, Muller H G and Agostini P 2001 Science 292 1689-1692

[12] Hentschel M, Kienberger R, Spielmann C, Reider G, Milosevic N, Brabec T, Corkum P, Heinzmann U, Drescher M and Krausz F 2001 Nature 414 509-513

[13] Zewail A H 2000 J. Phys. Chem. A 104 5660-5694

[14] Uiberacker M, Uphues T, Schultze M, Verhoef A, Yakovlev V, Kling M, Rauschenberger J, Kabachnik N, Schroder H, Lezius M, Kompa K, Muller H G, Vrakking M, Hendel S, Kleineberg U, Heinzmann U, Drescher M and Krausz F 2007 Nature 446 627-632

[15] Eckle P, Pfeiffer A N, Cirelli C, Staudte A, Dorner R, Muller H G, Buttiker M and Keller U 2008 Science 322 1525-1529

[16] Mauritsson J, Johnsson P, Mansten E, Swoboda M, Ruchon T, L'Huillier A and Schafer K 2008 Phys. Rev. Lett. 100073003

[17] Sola I, Mevel E, Elouga L, Constant E, Strelkov V, Poletto L, Villoresi P, Benedetti E, Caumes J P, Stagira S, Vozzi C, Sansone G and Nisoli M 2006 Nat Phys 2 319322

[18] Cavalieri A, Muller N, Uphues T, Yakovlev V, Baltuška A, Horvath B, Schmidt B, Blumel L, Holzwarth R, Hendel S, Drescher M, Kleineberg U, Echenique P, Kienberger R, Krausz F and Heinzmann U 2007 Nature 449 1029-1032

[19] Drescher M, Hentschel M, Kienberger R, Uiberacker M, Yakovlev V, Scrinzi A, Westerwalbesloh T, Kleineberg U, Heinzmann U and Krausz F 2002 Nature 419 803-807

[20] Föhlisch A, Feulner P, Hennies F, Fink A, Menzel D, Sanchez-Portal D, Echenique P M and Wurth W 2005 Nature 436 373-376

[21] Haessler S, Fabre B, Higuet J, Caillat J, Ruchon T, Breger P, Carré B, Constant E, Maquet A, Mével E, Salières P, Taïeb R and Mairesse Y 2009 Phys. Rev. A 80 011404(R)

[22] Swoboda M, Fordell T, Klünder K, Dahlström J M, Miranda M, Buth C, Schafer K J, Mauritsson J, L'Huillier A and Gisselbrecht M 2010 Phys. Rev. Lett. 104 103003

[23] Klünder K, Dahlström J M, Gisselbrecht M, Fordell T, Swoboda M, Guénot D, Johnsson P, Caillat J, Mauritsson J, Maquet A, Ta"ieb R and L'Huillier A 2011 Phys. Rev. Lett. 106143002

[24] Sansone G, Kelkensberg F, Perez-Torres J F, Morales F, Kling M F, Siu W, Ghafur O, Johnsson P, Swoboda M, Benedetti E, Ferrari F, Lepine F, Sanz-Vicario J L, Zherebtsov S, Znakovskaya I, L'Huillier A, Ivanov, Nisoli M, Martin F and Vrakking M J J 2010 Nature 465 763-766 
[25] Haessler S, Caillat J and Salières P 2011 J. Phys. B 44203001 ISSN 0953-4075

[26] Miyazaki K, Kaku M, Miyaji G, Abdurrouf A and Faisal 2005 Physical Review Letters 95243903

[27] Itatani J, Zeidler D, Levesque J, Spanner M, Villeneuve D M and Corkum P B 2005 Phys. Rev. Lett. 94123902

[28] Mairesse Y, Zeidler D, Dudovich N, Spanner M, Levesque J, Villeneuve D M and Corkum P B 2008 Phys. Rev. Lett. 100143903

[29] Baker S, Robinson J S, Haworth C A, Teng H, Smith R A, Chirilă C C, Lein M, Tisch J W G and Marangos J P 2006 Science 312 424-427

[30] Wagner N L, Wuest A, Christov I P, Popmintchev T, Zhou X, Murnane M M and Kapteyn H C 2006 PNAS USA 103 13279-13285

[31] Li W, Zhou X, Lock R, Patchkovskii S, Stolow A, Kapteyn H C and Murnane M M 2008 Science 322 1207-1211

[32] Haessler S, Boutu W, Stankiewicz M, Frasinski L J, Weber S, Caillat J, Taïeb R, Maquet A, Breger P, Monchicourt P, Carré B and Salières P 2009 J. Phys. B 42 134002

[33] Smirnova O, Mairesse Y, Patchkovskii S, Dudovich N, Villeneuve D, Corkum P and Ivanov M Y 2009 Nature 460 972-977

[34] Mairesse Y, Higuet J, Dudovich N, Shafir D, Fabre B, Mével E, Constant E, Patchkovskii S, Walters Z, Ivanov M Y and Smirnova O 2010 Phys. Rev. Lett. 104213601

[35] Schwarz W H E 2006 Angew. Chem. Int. Ed. 45 1508-1517

[36] Puschnig P, Berkebile S, Fleming A J, Koller G, Emtsev K, Seyller T, Riley J D, Ambrosch-Draxl C, Netzer F P and Ramsey M G 2009 Science 326 702-706

[37] Soe W H, Manzano C, De Sarkar A, Chandrasekhar N and Joachim C 2009 Phys. Rev. Lett. 102176102

[38] Gross L, Moll N, Mohn F, Curioni A, Meyer G, Hanke F and Persson M 2011 Phys. Rev. Lett. 107086101

[39] Wörner H J, Bertrand J B, Kartashov D V, Corkum P B and Villeneuve D M 2010 Nature 466 604-607

[40] Jackson J D 1998 Classical Electrodynamics (New York, USA: Wiley)

[41] Lewenstein M, Salières P and L'Huillier A 1995 Phys. Rev. A 52 4747-4754

[42] Ivanov M Y, Brabec T and Burnett N 1996 Phys. Rev. A 54 742-745

[43] Zwan E V and Lein M 2008 J. Phys. B 41074009

[44] Le A T, Della, Fainstein P, Telnov D, Lein M and Lin C 2008 J. Phys. B 41 081002

[45] Han Y C and Madsen L B 2010 Phy. Rev. A 81063430

[46] Chirilă C C and Lein M 2009 Phys. Rev. A 80013405 
[47] Friedrich B, Pullman D P and Herschbach D R 1991 J. Phys. Chem. 95 8118-8129

[48] Stapelfeldt H and Seideman T 2003 Rev. Mod. Phys. 75543

[49] Seideman $\mathrm{T}$ and Hamilton E 2005 Nonadiabatic Alignment by Intense Pulses. Concepts, Theory, and Directions vol 52 (Elsevier) pp 289-329 ISBN 9780120038527

[50] Kumarappan V, Viftrup S S, Holmegaard L, Bisgaard C Z and Stapelfeldt H 2007 Physica Scripta 76 C63

[51] Ghafur O, Rouzee A, Gijsbertsen A, Siu W K, Stolte S and Vrakking M J J 2009 Nature Physics 5 289-293

[52] Holmegaard L, Nielsen J H, Nevo I, Stapelfeldt H, Filsinger F, Küpper J and Meijer G 2009 Phys. Rev. Lett. 102023001

[53] Guerin S, Rouzee A and Hertz E 2008 Phys. Rev. A 77 041404(R)

[54] van der Zwan E V, Chirilă C C and Lein M 2008 Phys. Rev. A 78033410

[55] Salières P, Carré B, Le Deroff L, Grasbon F, Paulus G G, Walther H, Kopold R, Becker W, Milošević D B, Sanpera A and Lewenstein M 2001 Science 292 902-905

[56] Gaarde M, Tate J and Schafer K 2008 J. Phys. B 41132001

[57] Mairesse Y and Quéré F 2005 Phys. Rev. A 71011401

[58] Boutu W, Haessler S, Merdji H, Breger P, Waters G, Stankiewicz M, Frasinski L J, Taïeb R, Caillat J, Maquet A, Monchicourt P, Carré B and Salières P 2008 Nature Physics 4 545-549

[59] Mairesse Y, Dudovich N, Zeidler D, Spanner M, Villeneuve D M and Corkum P B 2010 J. Phys. B 43065401

[60] Zhou X, Lock R, Li W, Wagner N, Murnane M and Kapteyn H 2008 Phys. Rev. Lett. 100073902

[61] Lock R M, Zhou X, Li W, Murnane M M and Kapteyn H C 2009 Chemical Physics $36622-32$

[62] Wagner N, Zhou X, Lock R, Li W, Wuest A, Murnane M and Kapteyn H 2007 Phys. Rev. A 76061403

[63] McFarland B K, Farrell J P, Bucksbaum P H and Gühr M 2009 Phys. Rev. A 80 033412

[64] Kanai T, Takahashi E J, Nabekawa Y and Midorikawa K 2008 Phys. Rev. A 77 041402

[65] Levesque J, Mairesse Y, Dudovich N, Pepin H, Kieffer J, Corkum P B and Villeneuve D M 2007 Phys. Rev. Lett. 99243001

[66] Mairesse Y, Haessler S, Fabre B, Higuet J, Boutu W, Breger P, Constant E, Descamps D, Mével E, Petit S and Salières P 2008 New J. Phys. 10025028

[67] Zhou X, Lock R, Wagner N, Li W, Kapteyn H C and Murnane M M 2009 Phys. Rev. Lett. 102073902 
[68] Tate J, Auguste T, Muller H G, Salières P, Agostini P and DiMauro L F 2007 Phys. Rev. Lett. 98013901

[69] Colosimo P, Doumy G, Blaga C I, Wheeler J, Hauri C, Catoire F, Tate J, Chirla R, March A M, Paulus G G, Muller H G, Agostini P and DiMauro L F 2008 Nature Physics 4 386-389

[70] Takahashi E J, Kanai T, Ishikawa K L, Nabekawa Y and Midorikawa K 2008 Phys. Rev. Lett. 101253901

[71] Popmintchev T, Chen M C, Bahabad A, Gerrity M, Sidorenko P, Cohen O, Christov I P, Murnane M M and Kapteyn H C 2009 PNAS USA 10610516 10521

[72] Lein M, Hay N, Velotta R, Marangos J P and Knight P L 2002 Phys. Rev. A 66 023805

[73] Kamta L and Bandrauk A D 2005 Phys. Rev. A 71 053407-053419

[74] Kitzler M and Lezius M 2005 Phys. Rev. Lett. 95253001

[75] Kitzler M, Xie X, Scrinzi A and Baltuška A 2007 Phys. Rev. A 76011801

[76] Shafir D, Mairesse Y, Villeneuve D M, Corkum P B and Dudovich N 2009 Nature Physics 5 412-416

[77] Hijano E, Serrat C, Gibson G N and Biegert J 2010 Phys. Rev. A 81041401

[78] Le V H, Le A T, Xie R H and Lin C D 2007 Phys. Rev. A 76013413

[79] Walters Z B, Tonzani S and Greene C H 2008 J. Phys. Chem. A 112 9439-9447

[80] Wörner H J, Niikura H, Bertrand J B, Corkum P B and Villeneuve D M 2009 Phys. Rev. Lett. 102103901

[81] Higuet J, Ruf H, Thiré N, Cireasa R, Constant E, Cormier E, Descamps D, Mével E, Petit S, Pons B, Mairesse Y and Fabre B 2011 Phys. Rev. A 83053401

[82] Keldysh L V 1965 Sov. Phys. - JETP 20 1307-1314

[83] Tong X M, Zhao Z X and Lin C D 2002 Phys. Rev. A 66033402

[84] Kjeldsen T K and Madsen L B 2005 Phys. Rev. A 71023411

[85] Shiner A D, Schmidt B E, Trallero-Herrero C, Worner H J, Patchkovskii S, Corkum P B, Kieffer J C, Legare F and Villeneuve D M 2011 Nature Physics 7 464-467

[86] Lucchese R R, Raseev G and Mckoy V 1982 Phys. Rev. A 25 2572-2587

[87] Le A T, Lucchese R R, Tonzani S, Morishita T and Lin C D 2009 Phys. Rev. A 80013401

[88] van der Zwan E V and Lein M 2010 Phys. Rev. A 82033405

[89] Sukiasyan S, Patchkovskii S, Smirnova O, Brabec T and Ivanov M Y 2010 Physical Rev. A 82043414

[90] McFarland B K, Farrell J P, Bucksbaum P H and Gühr M 2008 Science 322 1232-1235 
[91] Lein M, Hay N, Velotta R, Marangos J P and Knight P L 2002 Phys. Rev. Lett. 88183903

[92] Bransden B H and Joachain C J 2003 Physics of Atoms and Molecules (Harlow, UK: Pearson Education)

[93] Lein M 2007 J. Phys. B 40 R135

[94] Ciappina M F, Chirilă C C and Lein M 2007 Phys. Rev. A 75 043405-043407

[95] Patchkovskii S, Zhao Z, Brabec T and Villeneuve D M 2007 J. Chem. Phys. 126 114306

[96] Le A T, Lucchese R R and Lin C D 2009 J. Phys. B 42211001

[97] Smirnova O, Patchkovskii S, Mairesse Y, Dudovich N and Ivanov M Y 2009 PNAS USA 106 16556-16561

[98] Torres R, Siegel T, Brugnera L, Procino I, Underwood J G, Altucci C, Velotta R, Springate E, Froud C, Turcu I C E, Patchkovskii S, Ivanov M Y, Smirnova O and Marangos J P 2010 Phys. Rev. A 81051802

[99] Farrell J P, Petretti S, Förster J, McFarland B K, Spector L S, Vanne Y V, Decleva P, Bucksbaum P H, Saenz A and Gühr M 2011 Phys. Rev. Lett. 107083001

[100] Kanai T, Takahashi E J, Nabekawa Y and Midorikawa K 2007 Phys. Rev. Lett. 98153904

[101] Kanai T, Minemoto S and Sakai H 2005 Nature 435 470-474

[102] Vozzi C, Calegari F, Benedetti E, Caumes J P, Sansone G, Stagira S, Nisoli M, Torres R, Heesel E, Kajumba N, Marangos J P, Altucci C and Velotta R 2005 Phys. Rev. Lett. 95 153902-153904

[103] Wörner H J, Bertrand J B, Hockett P, Corkum P B and Villeneuve D M 2010 Phys. Rev. Lett. 104233904

[104] Diveki Z, Camper A, Haessler S, Auguste T, Ruchon T, Carré B, Salières P, Guichard R, Caillat J, Maquet A and Tä̈eb R 2011 submitted

[105] Jordan G and Scrinzi A 2008 New J. Phys. 10025035

[106] Spanner M and Patchkovskii S 2009 Phys. Rev. A 80063411

[107] Walters Z B and Smirnova O 2010 J. Phys. B 43161002

[108] Sukiasyan S, McDonald C, Destefani C, Ivanov M Y and Brabec T 2009 Phys. Rev. Lett. 102223002

[109] Tchitchekova D S, Lu H, Chelkowski S and Bandrauk A D 2011 J. Phys. B 44 065601

[110] Caillat J, Zanghellini J, Kitzler M, Koch O, Kreuzer W and Scrinzi A 2005 Phys. Rev. A 71012712

[111] Patchkovskii S, Zhao Z, Brabec T and Villeneuve D M 2006 Phys. Rev. Lett. 97 123003-123004

[112] Santra R and Gordon A 2006 Phys. Rev. Lett. 96073906 
[113] Lünnemann S, Kuleff A I and Cederbaum L S 2009 J. Chem. Phys. 130154305

[114] Breidbach J and Cederbaum L S 2005 Phys. Rev. Lett. 94033901

[115] Hennig H, Breidbach J and Cederbaum L S 2005 J. Phys. Chem. A 109 409-414

[116] Smirnova O and Ivanov M 2010 Nature Physics 6 159-160

[117] Mairesse Y, de Bohan A, Frasinski L J, Merdji H, Dinu L C, Monchicourt P, Breger P, Kovacev M, Taïeb R, Carré B, Muller H G, Agostini P and Salières P 2003 Science 302 1540-1543

[118] Lein M 2005 Phys. Rev. Lett. 94053004

[119] Patchkovskii S 2009 Phys. Rev. Lett. 102253602

[120] Madsen C B, Samha M A and Madsen L B 2010 Phys. Rev. A 81043413

[121] Bredtmann T, Chelkowski S and Bandrauk A D 2011 Phys. Rev. A 84(2) 021401 URL http://link.aps.org/doi/10.1103/PhysRevA.84.021401

[122] Wörner H J, Bertrand J B, Fabre B, Higuet J, Ruf H, Dubrouil A, Patchkovskii S, Spanner M, Mairesse Y, Blanchet V, Mével E, Constant E, Corkum P B and Villeneuve D M 2011 Science 334208 